\title{
Application of Navy Color and Effects Model in Warship Virtual Scene Design
}

\author{
Ziwei Zhou ${ }^{1}$, Xueguang Zhou ${ }^{2, *}$ \\ ${ }^{1}$ Shanghai Design Institute, China Academy of Art, Shanghai, China \\ ${ }^{2}$ Department of Information Security, Naval University of Engineering, Wuhan, China
}

Email address:

1245790651@qq.com (Ziwei Zhou),zxg196610@hotmail.com (Xueguang Zhou)

*Corresponding author

\section{To cite this article:}

Ziwei Zhou, Xueguang Zhou. Application of Navy Color and Effects Model in Warship Virtual Scene Design. International Journal of Literature and Arts. Vol. 5, No. 2, 2017, pp. 7-12. doi: 10.11648/j.ijla.20170502.11

Received: April 9, 2017; Accepted: April 30, 2017; Published: May 16, 2017

\begin{abstract}
With the decreasing cost, VR technology has come back to hit of research again, the combination of it and warship mission virtual scene design has make it a new hot spot. This article gives three navy colors, which are the blue, the white and the grey, analyzes the ordinary special effect technology includes disaster spreading model, float condition model and vessel rolling model, conducts design work with naval color language and special effects model.
\end{abstract}

Keywords: Warship, VR, Scene Design, Science \& Art Combination, Naval Component

\section{Introduction}

Warship in the main platform to fulfill the mission of marine battle and target occupation, it is mobile territory of a country (which is entitled with diplomatic privileges and immunity when sailing or berthing on open waters and internal waters) and the key force to show the power. Chinese navy has advanced a lot since 2012. Plenty of new ships come out of the shipyard like dumplings dropping into the water, which has become the new normal, promoting the battle capability. Warships can be classified into battle vessels and auxiliary vessels in general, of which the former ones conduct tasks of battle while the later ones the logistical tasks like battle support, technology support and logistical support. A new vessel will go through 3 periods after coming into service, that is test \& trials, initial period and final period of battle power generation. The length of training time is related to the type of vessel, and the training tasks including vessel personnel training, equipment use training as well as the running and adjustment of the vessel. Vessel training can be classified into traditional vessel training practice and simulation digital training. This paper organizes and analyses the documents in 3D modeling, virtual demonstration and visual effect area, obtains the demonstration method of vessel and vessel missions [1-2] and then concludes the traditional culture and Chinese military science, applies VR and visual art to make the vessel digital information and demonstrate current value and show the China navy develop scene.

Since 2015, the operation cost of VR technology has decreased to an affordable level for ordinary commercial and civil enterprises, resulting in the outburst it in 2015, which is called "VR Year" [3]. VR has the factors of 3I: Immersion, Interaction and Imagination [4], which achieves the immersion of watching, interaction of vision, hearing, touch, thinking and behavior with the VR, through creating digital virtual scene. The core of VR is content construction, including cultural innovation, digital contents, comic and culture exhibition and so on. Since cutting-edge area like military and aerospace do not lack money, VR has been applied into these area once it has been proposed. Military training is to simulate the war procession with less investment and lower risk, which increases the training quality, shorten the developping interval, testify military theory and reduces training cost [5]. With the development of VR, this paper takes warship training task as the target, combines traditional tasks and modern VR technology and reconstructs training tasks and replays the training procession, emphasizes on the animation and digital media art and other methods, creates new VR contents in military area.

Mr. LI Zhengdao is an internationally renowned scientist, the winner of the Nobel prize. As he said in Science and 
Technology [6], art and science are both based on the creation of human, and persue the universality of truth. Art wakes the hidden, existing emotion in the human sense or subconsciousness with innovating methods. So the relationship of science and art is like wisdom and emotion, it tightly coupled. This article promotes transboundary talent cultivation through program research, which involves the vessel and marine subject 3D design. Special effects in VR art design includes vessel disaster spreading simulation, vessel floating condition model and rolling model, and afterwards processing. So this article involves technique innovation and culture creation, and extends the combination of science and art and tries transboundary talent cultivation.

In September 4, 2016, President Xi Jinping attended the opening ceremony of G20 summit in Hangzhou and said: "innovation is the growth of open lock fundamentally key, with the Internet as the core of a new round of technological and industrial revolution, gaining momentum, artificial intelligence, VR and other new technologies change rapidly".

Director of State Key Laboratory of VR Technology and System, the Chinese Academy of Engineering ZHAO Qin-ping is a leader in the field of VR in our country. He said VR has been into an era, the era of "VR+", which will promote the sustainable and healthy development of VR industry hand in hand. VR is a possible disruptive technologies, it will effect the future in 6 aspects: after personal computer, intelligent mobile phone, et al. VR is a new computing platform and environment, which will become the support platform for industry information technology development. VR has become the trend of the development in related industries, which will have to upgrade the development [7].

Navy is an international, strategic and comprehensive service. It is an important part of a state and army's international image, all forces spare no efforts to work on it. So enough attention should be paid to the navy image establishment. Display navy images vividly with vessel images, spread and build navy image in a direction that can be accepted by all. And the establishment of it cannot escape from the application of navy component, which includes marine sense, warship culture, fine tradition, navy color, science power, battle elements and so on. This article conduct research from application of navy color and design of VR special effects, and propose the design concept and method.

\section{Application of Navy Color in Virtual Scene Design}

Color is one of most important element in scene design. Once properly used it can strengthen the thought of the design work. The most typical color is navy. The thing which reflects sea as well as sky is sailor's striped shirt. It is blue and white, it is concise and clean. Wearing it gives the man the spirit and power of navy. The vessel equipped with shipboard aircraft also owns the cloud and sea. Blue can be divided into deep blue of deep water, and light blue of green water, sky blue of the sky. These all belong to the blue family culture. Only by applying blue when designing the scene can this work is related to the warships. Furthermore, blue is also the symbol of quietness, purity tonal contrast must also be taken in consideration, with one leading tonal decorated with a small part tonal, the work can be much better. Figure 1 is the VR picture of shipboard aircraft with 3D render.

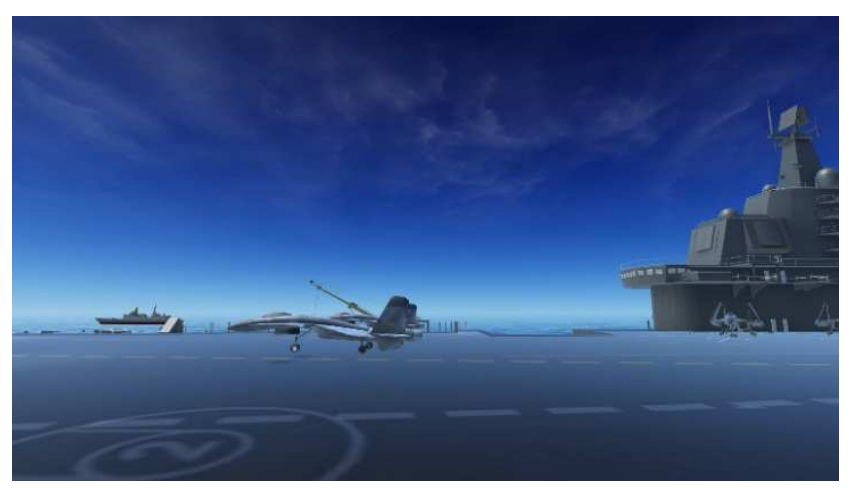

Figure 1. VR picture of shipboard aircraft.

The second navy color element is the typical color of grey, of which the image mark is metal, reflects the calmness, bored and insipid. Compared to the extern color of black and white, grey can easy the nerve. The visibility and occupationally of grey is rather low, which makes the grey rather compliant. The mentality character of grey is very casual and indifferent in some conditions. So the application of grey can soften the traditionalist of form and promote the general feel of the picture. And in terms of the match of colors, application of grey can promote the sense of fashion and make the picture more harmonious. Figure 2 is the taking off picture in VR helmet vision.

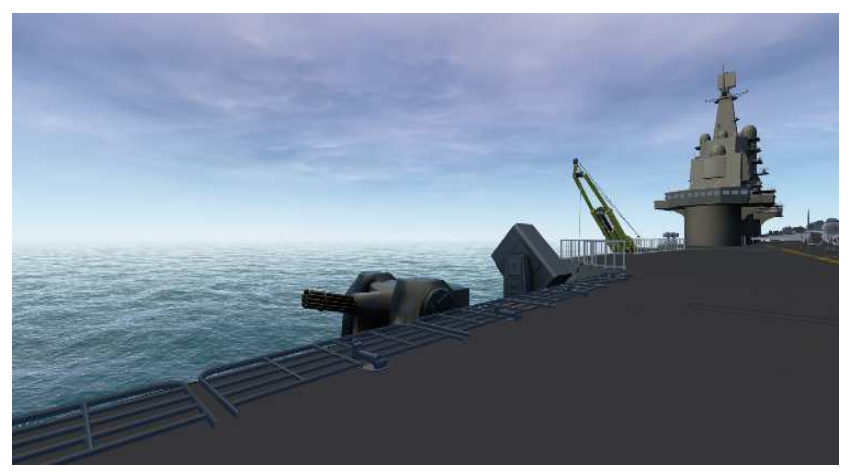

Figure 2. Shipboard aircraft taking off in the vision of VR helmet.

Oculus Rift is a head mounted display (HMD), which is released in 2013 by VR technology manufacturers and acquired by Facebook in 2014 at a high price. Mark Zuckerberg, the founder and the CEO of Fackbook thinks that Oculus has achieved success in immersive virtual game and he is confident to extend the existing advantages of this technology to communication, media, education and other new vertical fields, and build the largest social network platform to change people's work, communication and games. Figure 3 show the shipboard aircraft of our VR work viewed in the Oculus helmet. 


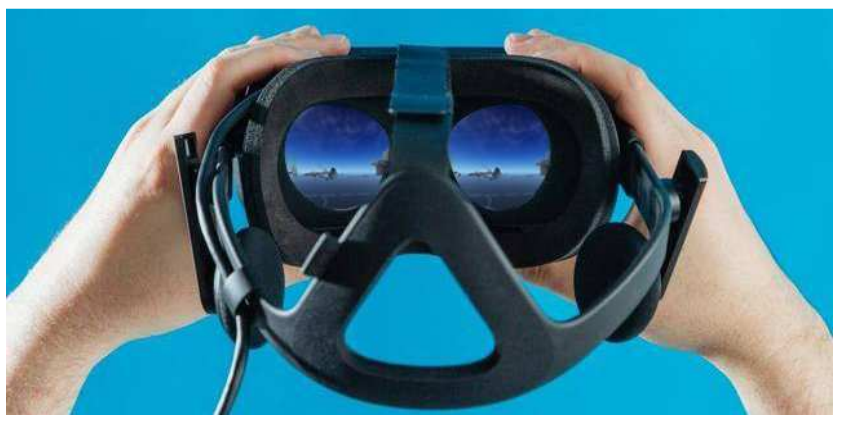

Figure 3. VR picture of peaceful vessel deck.

\section{Application of Warship Special Effect Model in Virtual Scene Design}

Special effects refer to the phenomenon that cannot be controlled in the real life, which can be simulated and created by computer and always follow the human's will on the contrary. It's widely used in military exercises, video production, game creation and other fields. There are a lot of cases in which special effects promote the artistic expression. Warship virtual scene design cannot avoid using it. The common vessel special effects include: disaster spread model [8], float posture model [9] and vessel rolling model [10].

\subsection{Disaster Spreading Model}

To make the $3 \mathrm{D}$ display of virtual scene identified to the reality, the water incoming speed, water incoming amount, fire spreading speed, smoke spreading speed and other factors should be built into function curve to be displayed as highest driven level code. The computation model of incoming water spreading is built on following conditions satisfied: all the curve of broken area must satisfy the Bernoulli equation. All the leaking cabins must satisfy incoming water balance equation, that is the total incoming flow in the crevasse must equal to the incoming flow of the cabin at any time. Damaged vessels should satisfy the posture balance equations.

When build the posture index model of leaking process, we assume that: Vessel should be static balanced, the water level the ship should be along with the sea level and lower than the sea level at any time in the leaking period. There is no delay of water flow when the pressure difference is generated. Considering the cabin water level changing speed and crevasse water flow speed are not much different, the influence of the difference is ignored: the flow speed of crevasse is only related to the level difference, crevasse area and flow index. The flow index of crevasse does not differ with the changing of water level difference.

Figure 4 and Figure 5 are the changing relationship of the cabin water level and vessel minimum freeboard during the leak.

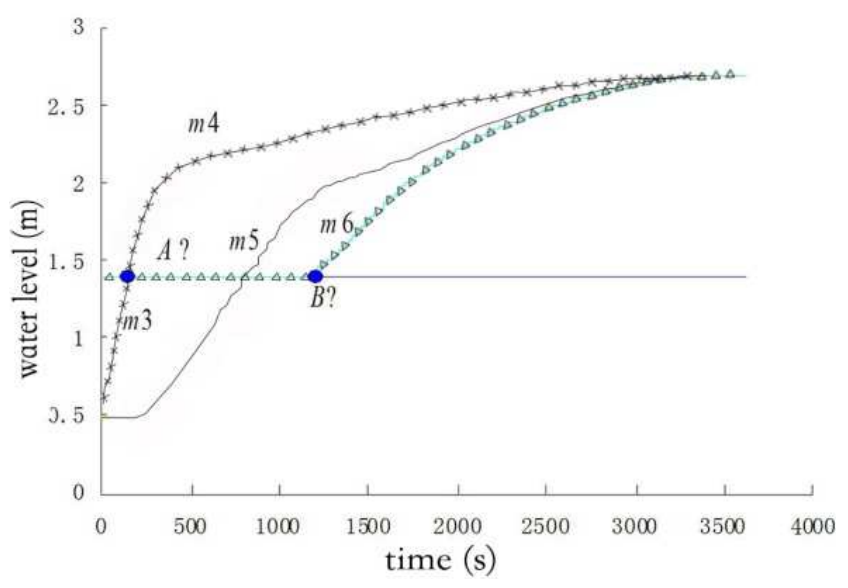

Figure 4. Water level-time changing curve.

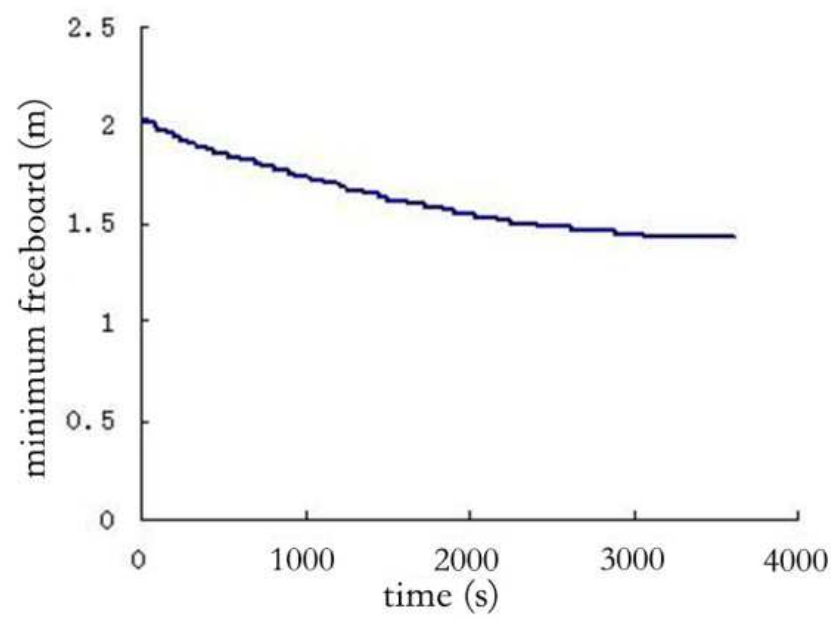

Figure 5. Minimum freeboard-time changing curve.

The fire spread model is presented in Figure 6. The fire double-deck area simulation method is used, that is to dive the space into upper smoke layer and lower air layer.

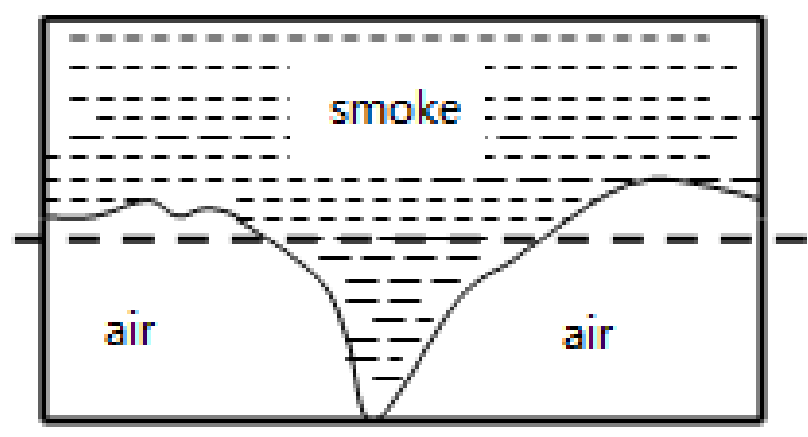

Figure 6. Cabin fire model.

And to assume that the physical parameter in every area is uniformed and according, the energy exchange between areas is conducted by fir plume, and a group of ordinary differential control equation is obtained with law of conservation of mass, law of conservation of energy, gas state equation, law of thermodynamics and other laws. There is no direct energy, power exchanges between areas, see figure 7(a) and 7(b). The smoke plume takes the $70 \%$ energy of fire and the rest work on heating lower air and boundary. 

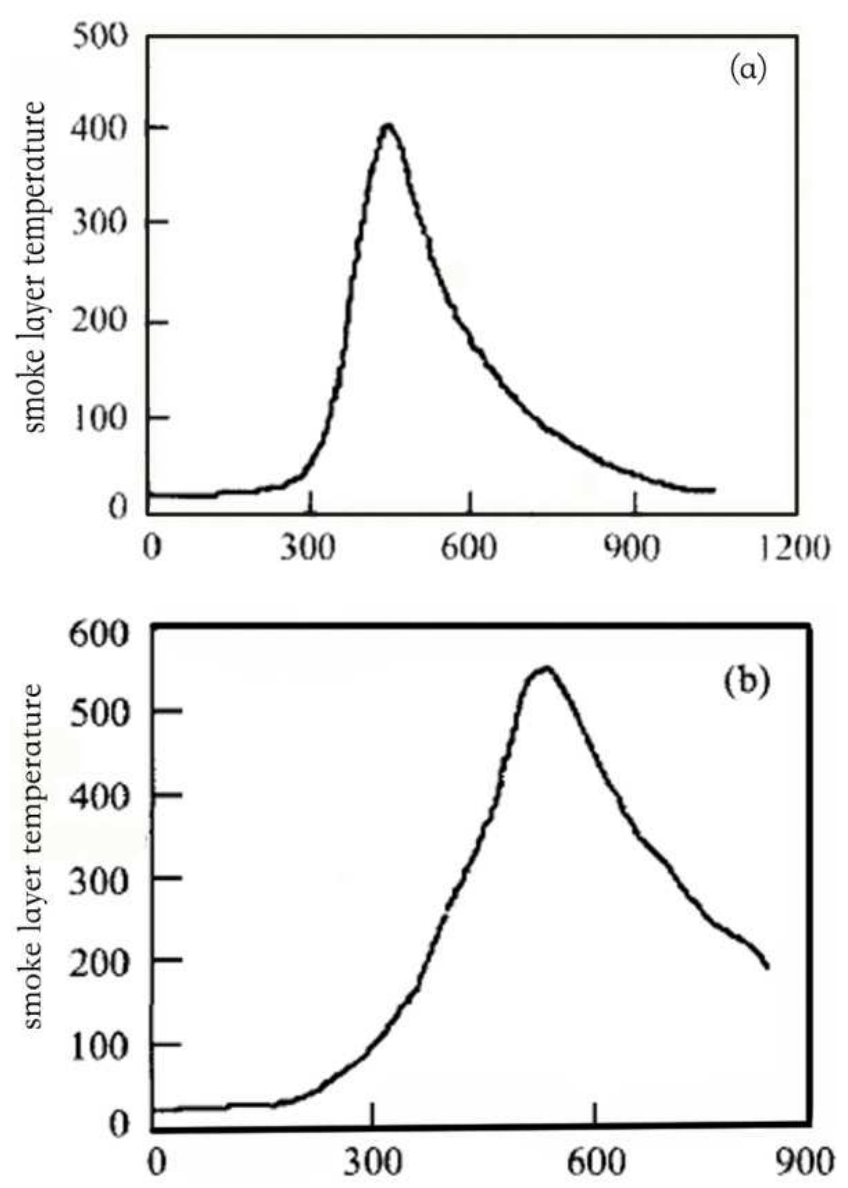

Figure 7. Computation case of smoke layer temperature.

\subsection{Warship Floating Posture Model}

When the cabin is damaged and leaking or there is water after fire control, influence will be cast to the floating posture model. Through this method we can simulate the change of vessel posture and support the visualization of outer direction.

Vessel is balanced at any time of the water spreading. We only consider the draft changing, heel and trim but not the upper and lower, rolling and pitch. So the vessel must obey the equations below.

$$
\left\{\begin{array}{l}
\bar{V}\left(T_{m}, \theta, \phi, V_{c i}\right)-V_{0}=0 \\
M_{z x}\left(T_{m}, \theta, \phi, V_{c i}\right)+M_{x y}\left(T_{m}, \theta, \phi, V_{c i}\right) \operatorname{tg} \theta=0 \\
M_{y z}\left(T_{m}, \theta, \phi, V_{c i}\right)+M_{x y}\left(T_{m}, \theta, \phi, V_{c i}\right) \operatorname{tg} \phi=0
\end{array}\right.
$$

$V_{c i}$ is the water volume of cabin $i ; \bar{V}\left(T_{m}, \theta, \varphi, V_{c i}\right)$ is the valid draining volume; $\mathrm{V}_{0}$ is the primary draining volume; $M_{z x}\left(T_{m}, \theta, \varphi, V_{c i}\right)$ is the horizontal volume static moment of vessel to planeZOX; $M_{y z}\left(T_{m}, \theta, \varphi, V_{c i}\right)$ is the longitudinal volume static moment of vessel to plane $Y O Z$; $M_{x y}\left(T_{m}, \theta, \varphi, V_{c i}\right)$ is the vertical volume static moment of vessel to plane $X O Y$.

\subsection{The Rolling Model of Vessel Sailing in Waves}

We conduct simulation of posture and rules of rolling.

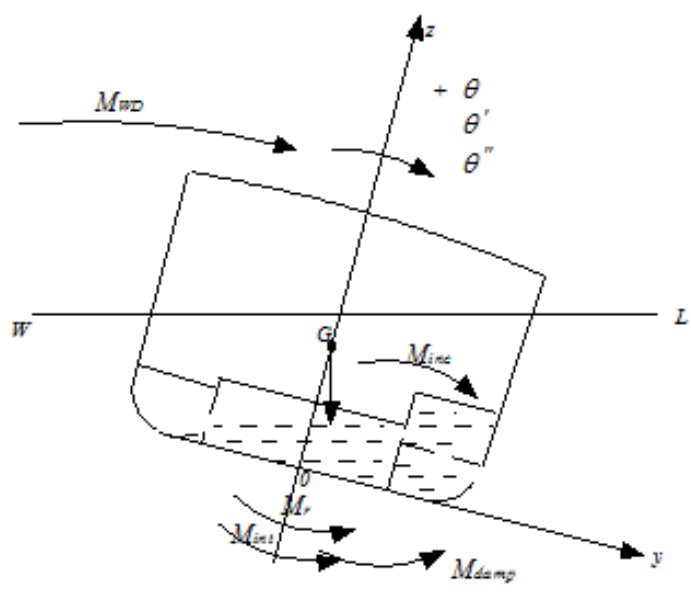

Figure 8. SRESS during the rolling procession.

The calculation of dynamic stability when the ship is rolling must satisfy the following equations: rolling movement equation, vertical stress balance equation, and vertical balance equation.
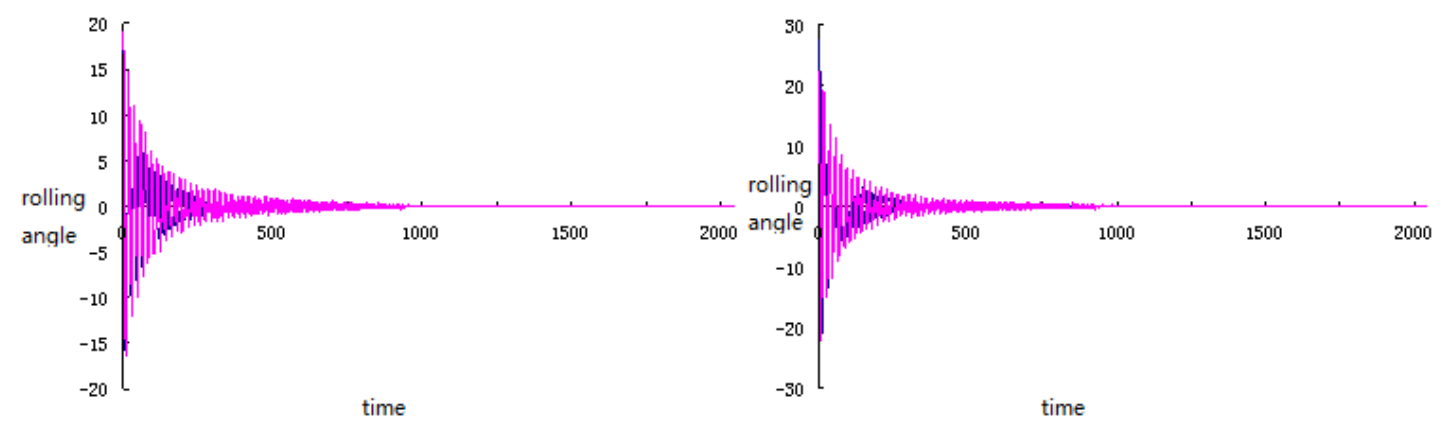

Figure 9. Computation case of rolling angle.

\subsection{Display of Virtual Scene}

According to the leaking, fire and smoke spreading process calculated above, we achieve visualization with comprehensive use of OpenGL, 3Dmax, Flash and other tools.

Figure 10 shows the profile of vessel cabin interior after the fire. It is located at the bottom of the vessel. 


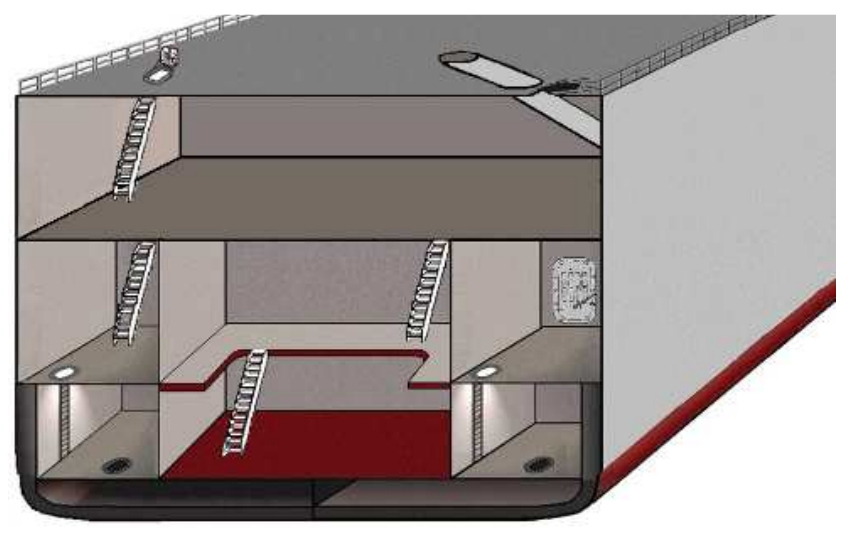

Figure 10. Inner structure of cabin.

Figure 11 gives a glimpse of the effect of the vessel compartment after the fire spread to other layers of the map, which can be referred to in the fire fighting or to make the rescue plan.
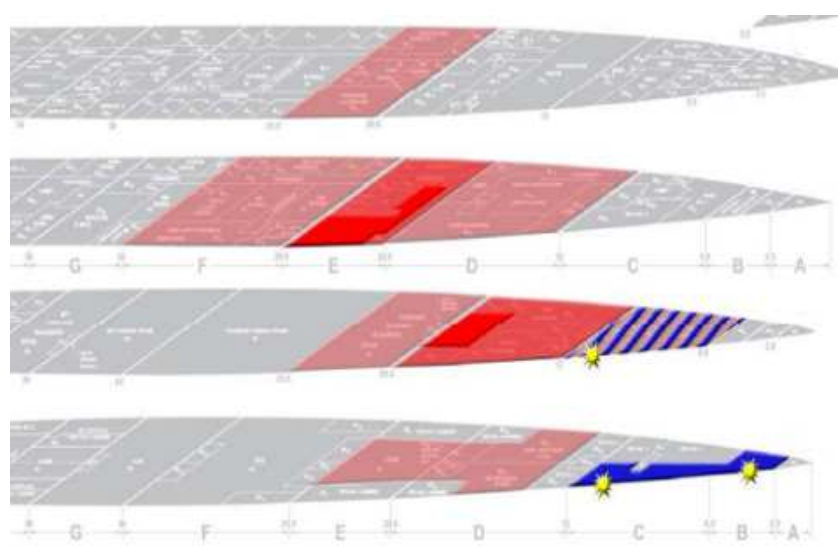

Figure 11. Disaster spreading context distribution.

Figure 12 shows the rescue workers in the preparation.

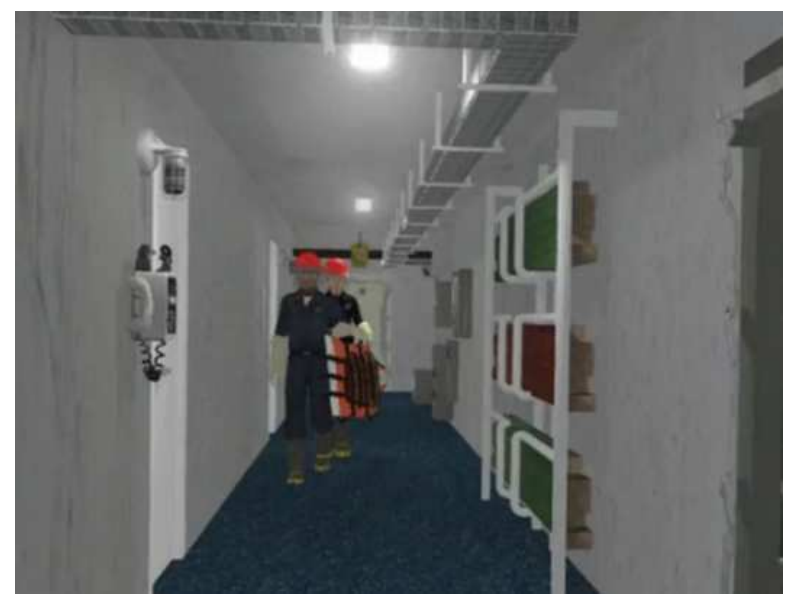

Figure 12. Battle position damage control model.

\section{Conclusion}

VR involves the industrial design, military science, psychology, communications and computer science. It enjoys great integrity and intersection. Now it has become the 3rd method in the science exploration other than theoretically study and scientifically experiment. This article analyzes the consistency of navy components, proposes that blue, white and grey is the stereotype of navy color and designs some vessel VR with them. Then 3 vessel special effects are proposed: disaster spread model, float posture model and the vessel rolling model, which are used to direct and realize the design of some virtual warship scenes.

ZHAO Qin-ping, the academician of Chinese Academy of Engineering, thinks that there are two directions of VR development in the future: one is mass consumption; the other is the industry application. So our next work is to emphasize the study of VR content narrative and reconstruction, bring the best of the interaction, immersion and imagination, and make more and better VR works.

\section{Acknowledgements}

The authors would likely to give thanks to anonymous reviewers for the comments and suggestions. We would also like to thank the national natural science foundation of China. This paper is part of our foundation work, the grant number is 61672531

\section{References}

[1] ZHAO Qinping. Survey on Virtual Reality. Science in China. F: Information Science. 2009,39(1):2-46(in Chinese)

[2] ZHAO Qinping, ZHOU Bin, LI Jia, CHEN Xiaowu. A Brief Survey on virtual Reality Technology. Science \& Technology Review, 2016,34(14):71-75(in Chinese)

[3] WANG Tao, DUAN Youqiao. Key Technologies and Development of VR Panoramic Video, Communications of the CCF, 2016,12(12):42-49(in Chinese)

[4] Burdea G, Coiffet P. Virtual Reality Technology[M].NJ: John Wiley and Sons, 1994

[5] [EB/OL].http://m.sohu.com/n/467248337/.2017-01-27

[6] LI Zhengdao. Science and technology. Tianjin Science \& Technology. 2004,(6):38(in Chinese)

[7] ZHAO Qinping, JIANG Kai. The eve of the outbreak of the VR industry. Scientia Sinica (Informationis), 2016, 46(12): 1774-1778(in Chinese)

[8] Hongpo WANG, Hong ZHOU. A Fire Spread Model Based on Cellular Automaton. International Conference on Soft Computing in Information Communication Technology (SCICT2014).Taipei, Taiwan, China. May 31, 2014. Atlantic Press. 191-194.

[9] ZENG Xiaofan, CAI weijun, XU Xindong, WANG Gaidi, BIAN Xiang. Analysis and Engineering Calculation of Torpedo Floating Attitude at Running End. Torpedo Technology. 2014, 22(5): 325-328.(in Chinese)

[10] DU Jianping. The Analysis on the Ship's Capsizing in Following Seas. [Dissertation to the Master of Engineering] Dalian: Dalian Maritime University. 2014.5-6.(in Chinese) 


\section{Biography}

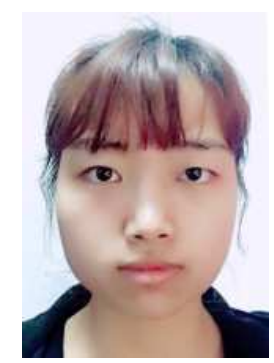

Ziwei Zhou, is currently a Master Degree Candidate of design science at the Shanghai Design Institute, China Academy of Art, Shanghai, China. Her current research interests include design and implementation of virtual reality, narrative and reconstruction of virtual presentation.

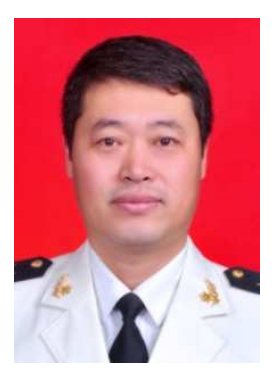

Xueguang Zhou, the corresponding author, e-mail: zxg196610@hotmail.com. He received his $\mathrm{PhD}$ information security from Wuhan University. He is a professor and $\mathrm{PhD}$ supervisor of the Department of Information Security, Naval University of Engineering. $\mathrm{He}$ is also a Senior Member of China Computer Federation. His current research interests include information content security, social network analysis and virtual reality. 